\title{
The Properties of Various Concepts
}

\author{
Yong Chan Kim and Jung Mi Ko \\ Department of Mathematics, Gangneung-Wonju National University, Gangneung, 201-702, Korea
}

\begin{abstract}
In this paper, we investigate the relations among formal concepts, attribute oriented concept and object oriented concepts on a complete residuated lattice.

Key Words : Complete residuated lattices, order reverse generating maps, isotone (antitone) Galois connetion, formal (resp. attribute oriented, object oriented) concepts
\end{abstract}

\section{Introduction and preliminaries}

Wille [11] introduced the formal concept lattices by allowing some uncertainty in data. Formal concept analysis is an important mathematical tool for data analysis and knowledge processing [1-5,8,9,11]. A fuzzy context consists of $(X, Y, R)$ where $X$ is a set of objects, $Y$ is a set of attributes and $R$ is a relation between $X$ and $Y$. Bělohlávek [1-4] developed the notion of formal concepts with $R \in L^{X \times Y}$ on a complete residuated lattice $L$.

In this paper, we show that the classes of formal concepts, attribute oriented concept and object oriented concepts are complete lattices. Moreover, a family of attribute oriented concepts and a family of object oriented concepts are anti-isomorphic, a family of attribute oriented concepts and a family of formal concepts are isomorphic, a family of object oriented concepts and a family of formal concepts are anti-isomorphic.

Definition 1.1. $[1-5,10]$ A triple $(L, \leq, \odot)$ is called a complete residuated lattice iff it satisfies the following conditions:

(L1) $L=(L, \leq, 1,0)$ is a complete lattice where 1 is the universal upper bound and 0 denotes the universal lower bound;

(L2) $(L, \odot, 1)$ is a commutative monoid;

(L3) $\odot$ is distributive over arbitrary joins, i.e.

$$
\left(\bigvee_{i \in \Gamma} a_{i}\right) \odot b=\bigvee_{i \in \Gamma}\left(a_{i} \odot b\right)
$$

Define an operation $\rightarrow$ as $a \rightarrow b=\bigvee\{c \in L \mid a \odot c \leq$ $b\}$, for each $a, b \in L$.

Let $(L, \leq, \odot)$ be a complete residuated lattice. A order reversing map * $: L \rightarrow L$ defined by $a^{*}=a \rightarrow 0$ is called a strong negation if $a^{* *}=a$ for each $a \in L$.

In this paper, we assume $\left(L, \leq, \odot,^{*}\right)$ is a complete residuated lattice with a strong negation ${ }^{*}$.

Manuscript received Dec. 24. 2009; revised . Mar. 6, 2010; arcepted Sep. 5, 2010;
Definition 1.2. [5,8,9] Let $X$ and $Y$ be two sets. Let $\omega \rightarrow, \phi^{\rightarrow}, \xi^{\rightarrow}: L^{X} \rightarrow L^{Y}$ and $\omega^{\leftarrow}, \phi^{\leftarrow}, \xi^{\leftarrow}: L^{Y} \rightarrow L^{X}$ be operators.

(1) The pair $\left(\omega^{\rightarrow}, \omega^{\leftarrow}\right)$ is called antitone Galois connection between $X$ and $Y$ if for $\mu \in L^{X}$ and $\rho \in L^{Y}$, $\rho \leq \omega^{\rightarrow}(\mu)$ iff $\mu \leq \omega^{\leftarrow}(\rho)$.

(2) The pair $\left(\phi^{\rightarrow}, \phi^{\leftarrow}\right)$ is called an isotone Galois connection between $X$ and $Y$ if for $\mu \in L^{X}$ and $\rho \in L^{Y}$, $\phi^{\rightarrow}(\mu) \leq \rho$ iff $\mu \leq \phi^{\leftarrow}(\rho)$. Moreover, the pair $\left(\xi^{\leftarrow}, \xi^{\rightarrow}\right)$ is called an isotone Galois connection between $X$ and $Y$ if for $\mu \in L^{X}$ and $\rho \in L^{Y}, \xi^{\leftarrow}(\rho) \leq \mu$ iff $\rho \leq \xi^{\rightarrow}(\mu)$.

Definition 1.3. [5,8,9] Let $\omega^{\rightarrow}, \phi \rightarrow, \xi \rightarrow: L^{X} \rightarrow L^{Y}$ and $\omega^{\leftarrow}, \phi^{\leftarrow}, \xi^{\leftarrow}: L^{Y} \rightarrow L^{X}$ be functions. A pair $(\mu, \rho) \in L^{X} \times L^{Y}$ is called:

(1) formal concept if $\rho=\omega^{\rightarrow}(\mu)$ and $\mu=\omega^{\leftarrow}(\rho)$ where $\left(\omega^{\rightarrow}, \omega^{\leftarrow}\right)$ is an antitone Galios connection,

(2) an attribute oriented concept if $\rho=\phi \rightarrow(\mu)$ and $\mu=\phi^{\leftarrow}(\rho)$ where $\left(\phi^{\rightarrow}, \phi^{\leftarrow}\right)$ is an isotone Galios connection,

(3) an object oriented concept if $\rho=\xi \rightarrow(\mu)$ and $\mu=\xi^{\leftarrow}(\rho)$ where $\left(\xi^{\leftarrow}, \xi^{\rightarrow}\right)$ is an isotone Galios connection.

Theorem 1.4. [9] Let $\omega^{\rightarrow}: L^{X} \rightarrow L^{Y}$ and $\omega^{\leftarrow}: L^{Y} \rightarrow$ $L^{X}$ be operators. Let $\left(\omega^{\rightarrow}, \omega^{\leftarrow}\right)$ be an antitone Galois connection between $X$ and $Y$. Then the following properties hold:

(1) For each $\mu \in L^{X}$ and $\mu \in L^{X}, \mu \leq \omega^{\leftarrow}(\omega \rightarrow(\mu))$ and $\rho \leq \omega^{\rightarrow}\left(\omega^{\leftarrow}(\rho)\right)$.

(2) If $\mu_{1} \leq \mu_{2}$, then $\omega^{\rightarrow}\left(\mu_{1}\right) \geq \omega^{\rightarrow}\left(\mu_{2}\right)$. Moreover, if $\rho_{1} \leq \rho_{2}$, then $\omega^{\leftarrow}\left(\rho_{1}\right) \geq \omega^{\leftarrow}\left(\rho_{2}\right)$.

(3) For each $\mu \in L^{X}$ and $\rho \in L^{Y}$, $\omega^{\leftarrow}\left(\omega^{\rightarrow}\left(\omega^{\leftarrow}(\rho)\right)\right)=\omega^{\leftarrow}(\rho)$ and $\omega^{\rightarrow}\left(\omega^{\leftarrow}\left(\omega^{\rightarrow}(\mu)\right)\right)=$ $\omega \rightarrow(\mu)$.

(4) For each $\mu_{i} \in L^{X}$ and $\rho_{j} \in L^{Y}, \omega \rightarrow\left(\bigvee_{i \in I} \mu_{i}\right)=$ $\bigwedge_{i \in I} \omega^{\rightarrow}\left(\mu_{i}\right)$ and $\omega^{\leftarrow}\left(\bigvee_{j \in J} \rho_{j}\right)=\bigwedge_{j \in J} \omega^{\leftarrow}\left(\rho_{j}\right)$. 
Theorem 1.5. [8] Let $\phi^{\rightarrow}: L^{X} \rightarrow L^{Y}$ and $\phi^{\leftarrow}: L^{Y} \rightarrow$ $L^{X}$ be operators. Let $\left(\phi^{\rightarrow}, \phi^{\leftarrow}\right)$ be an isotone Galois connection between $X$ and $Y$. For each $\mu, \mu_{i} \in L^{X}$ and $\rho, \rho_{j} \in L^{Y}$, the following properties hold:

(1) $\mu \leq \phi^{\leftarrow}\left(\phi^{\rightarrow}(\mu)\right)$ and $\phi^{\rightarrow}\left(\phi^{\leftarrow}(\rho)\right) \leq \rho$.

(2) If $\mu_{1} \leq \mu_{2}$, then $\phi \rightarrow\left(\mu_{1}\right) \leq \phi \rightarrow\left(\mu_{2}\right)$. Moreover, if $\rho_{1} \leq \rho_{2}$, then $\phi^{\leftarrow}\left(\rho_{1}\right) \leq \phi^{\leftarrow}\left(\rho_{2}\right)$.

(3) $\phi^{\leftarrow}\left(\phi^{\rightarrow}\left(\phi^{\leftarrow}(\rho)\right)\right)=\phi^{\leftarrow}(\rho)$ and $\phi^{\rightarrow}\left(\phi^{\leftarrow}\left(\phi^{\rightarrow}(\mu)\right)\right)=$ $\phi \rightarrow(\mu)$.

(4) $\phi \rightarrow\left(\bigvee_{i \in I} \mu_{i}\right) \quad=\quad \bigvee_{i \in I} \phi^{\rightarrow}\left(\mu_{i}\right) \quad$ and $\phi^{\leftarrow}\left(\bigwedge_{j \in J} \rho_{j}\right)=\bigwedge_{j \in J} \phi^{\leftarrow}\left(\rho_{j}\right)$.

Definition 1.6. [8,9] An operator $\phi^{\rightarrow}: L^{X} \rightarrow L^{Y}$ is called a join-generating operator, denoted by $\phi^{\rightarrow} \in J(X, Y)$, if $\phi \rightarrow\left(\bigvee_{i \in \Gamma} \lambda_{i}\right)=\bigvee_{i \in \Gamma} \phi^{\rightarrow}\left(\lambda_{i}\right)$, for $\left\{\lambda_{i}\right\}_{i \in \Gamma} \subset L^{X}$.

An operator $\psi^{\rightarrow}: L^{X} \rightarrow L^{Y}$ is called a meetgenerating operator, denoted by $\psi \rightarrow \in M(X, Y)$, if $\psi \rightarrow\left(\bigwedge_{i \in \Gamma} \lambda_{i}\right)=\bigwedge_{i \in \Gamma} \psi \rightarrow\left(\lambda_{i}\right)$, for $\left\{\lambda_{i}\right\}_{i \in \Gamma} \subset L^{X}$.

An operator $\omega^{\rightarrow}: L^{X} \rightarrow L^{Y}$ is called a order reverse-generating operator, denoted by $\omega \rightarrow \in K(X, Y)$, if $\omega \rightarrow\left(\bigvee_{i \in \Gamma} \lambda_{i}\right)=\bigwedge_{i \in \Gamma} \omega \rightarrow\left(\lambda_{i}\right)$, for $\left\{\lambda_{i}\right\}_{i \in \Gamma} \subset L^{X}$.

Theorem 1.7. [9] For $\omega \rightarrow \in K(X, Y)$, Define functions $\phi_{\omega}, \xi_{\omega}: L^{X} \rightarrow L^{Y}$ and $\omega^{\leftarrow}, \phi_{\omega}^{\leftarrow}, \xi_{\omega}^{\leftarrow}: L^{Y} \rightarrow L^{X}$ as follows: for all $\lambda \in L^{X}, \rho \in L^{Y}$,

$$
\begin{gathered}
\omega^{\leftarrow}(\rho)=\bigvee\left\{\lambda \in L^{X} \mid \omega^{\rightarrow}(\lambda) \geq \rho\right\} \\
\phi_{\omega}^{\rightarrow}(\mu)=\left(\omega^{\rightarrow}(\mu)\right)^{*}, \phi_{\omega}^{\leftarrow}(\rho)=\omega^{\leftarrow}\left(\rho^{*}\right) \\
\xi_{\omega}^{\leftarrow}(\rho)=\bigwedge\left\{\lambda \in L^{X} \mid \omega\left(\lambda^{*}\right) \geq \rho\right\}, \\
\xi_{\omega}^{\rightarrow}(\mu)=\bigvee\left\{\rho \in L^{Y} \mid \xi_{\omega}^{\leftarrow}(\rho) \leq \mu\right\}
\end{gathered}
$$

Then the following properties hold:

(1) $\omega^{\leftarrow} \in K(Y, X)$ with $\omega^{\rightarrow}(\lambda) \geq \rho \Leftrightarrow \omega^{\leftarrow}(\rho) \geq \lambda$ for all $\lambda \in L^{X}$ and $\rho \in L^{Y}$.

(2) The pair $\left(\omega^{\rightarrow}, \omega^{\leftarrow}\right)$ is an antitone Galois connection and $\left(\omega^{\leftarrow}\left(\omega^{\rightarrow}(\lambda)\right), \omega^{\rightarrow}(\lambda)\right)$ for all $\lambda \in L^{X}$ are formal concepts.

(3) The pair $\left(\phi_{\omega}, \phi_{\omega}^{\leftarrow}\right)$ is an isotone Galois connection and $\left(\phi_{\omega}^{\leftarrow}\left(\phi_{\omega}(\lambda)\right), \phi_{\omega}(\lambda)\right)=\left(\omega^{\leftarrow}\left(\omega^{\rightarrow}(\lambda)\right), \omega^{\rightarrow}(\lambda)^{*}\right)$ for all $\lambda \in L^{X}$ are attribute oriented concepts.

(4) $\xi_{\omega}^{\leftarrow}: L^{Y} \rightarrow L^{X}$ is a join-generating function such that $\xi_{\omega}^{\leftarrow}(\rho)=\left(\omega^{\leftarrow}(\rho)\right)^{*}$ and $\xi_{\omega}: L^{X} \rightarrow L^{Y}$ is a meetgenerating function such that $\xi_{\omega}(\lambda)=\omega \rightarrow\left(\lambda^{*}\right)$.

(5) The pair $\left(\xi_{\omega}^{\leftarrow}, \xi_{\omega}\right)$ is an isotone Galois connection and $\left(\xi_{\omega}^{\leftarrow}(\rho), \xi_{\omega}\left(\xi_{\omega}^{\leftarrow}(\rho)\right)\right)=\left(\omega^{\leftarrow}(\rho)^{*}, \omega^{\rightarrow}\left(\omega^{\rightarrow}(\rho)\right)\right)$ for all $\rho \in L^{Y}$ are object oriented concepts.

Theorem 1.8. [9] Let $(X, Y, R)$ be a fuzzy context. Define a function $\omega_{R}: L^{X} \rightarrow L^{Y}$ as follows:

$$
\omega_{R}(\lambda)(y)=\bigvee_{x \in X}(\lambda(x) \rightarrow R(x, y))
$$

Then we have the following properties:
(1) $\omega_{R} \rightarrow K(X, Y),\left(\omega_{R}, \omega_{R}^{\leftarrow}\right)$ is an isotone Galois connections and $\left(\omega_{R}^{\leftarrow}\left(\omega_{R}(\lambda)\right), \omega_{R}(\lambda)\right)$ for all $\lambda \in L^{X}$ are formal concepts where

$$
\omega_{R}^{\leftarrow}(\rho)(x)=\bigwedge_{y \in Y}(\rho(y) \rightarrow R(x, y))
$$

(2) $\phi_{\omega_{R}}(\mu)=\left(\omega_{R}(\mu)\right)^{*}$ and $\phi_{\omega_{R}}^{\leftarrow}(\rho)=\omega_{R}^{\overleftarrow{R}}\left(\rho^{*}\right)$ where

$$
\begin{aligned}
& \phi_{\omega_{R}}^{\rightarrow}(\mu)(y)=\bigvee_{y \in Y}\left(\mu(x) \odot R^{*}(x, y)\right), \\
& \phi_{\omega_{R}}^{\leftarrow}(\rho)(x)=\bigwedge_{y \in Y}\left(R^{*}(x, y) \rightarrow \rho(y)\right) .
\end{aligned}
$$

Moreover, the pair $\left(\phi_{\omega_{R}}, \phi_{\omega_{R}}^{\leftarrow}\right)$ is an isotone Galois connection and $\left(\phi_{\omega_{R}}^{\leftarrow}\left(\phi_{\omega_{R}}(\lambda)\right), \phi_{\omega_{R}}(\lambda)\right)$ are attribute concepts.

(3) The pair $\left(\xi_{\omega_{R}}^{\leftarrow}, \xi_{\omega_{R}}\right)$ is an isotone Galois connection and $\left(\xi_{\omega_{R}}^{\leftarrow}(\rho), \xi_{\omega_{R}}\left(\xi_{\omega_{R}}^{\leftarrow}(\rho)\right)\right)$ for all $\rho \in L^{Y}$ are object oriented concepts where

$$
\begin{aligned}
& \xi_{\omega_{R}}^{\leftarrow}(\rho)(x)=\bigvee_{y \in Y}\left(\rho(y) \odot R^{*}(x, y)\right), \\
& \xi_{\omega_{R}}^{\rightarrow}(\lambda)(y)=\bigwedge_{x \in X}\left(R^{*}(x, y) \rightarrow \lambda(x)\right)
\end{aligned}
$$

Theorem 1.9. [8] For $\phi \rightarrow \in J(X, Y)$, Define functions $\omega_{\phi}, \xi_{\phi}: L^{X} \rightarrow L^{Y}$ and $\phi^{\leftarrow}, \omega_{\phi}^{\leftarrow}, \xi_{\phi}^{\leftarrow}: L^{Y} \rightarrow L^{X}$ as follows: , for all $\lambda \in L^{X}, \rho \in L^{Y}$,

$$
\begin{gathered}
\phi^{\leftarrow}(\rho)=\bigvee\left\{\lambda \in L^{X} \mid \phi^{\rightarrow}(\lambda) \leq \rho\right\}, \\
\omega_{\phi}^{\rightarrow}(\lambda)=\left(\phi^{\rightarrow}(\lambda)\right)^{*}, \quad \omega_{\phi}^{\leftarrow}(\rho)=\phi^{\leftarrow}\left(\rho^{*}\right), \\
\xi_{\phi}^{\leftarrow}(\rho)=\bigwedge\left\{\lambda \in L^{X} \mid \phi^{\rightarrow}\left(\lambda^{*}\right) \leq \rho^{*}\right\}, \\
\xi_{\phi}(\lambda)=\bigvee\left\{\rho \in L^{Y} \mid \xi_{\phi}^{\leftarrow}(\rho) \leq \lambda\right\} .
\end{gathered}
$$

Then the following properties hold:

(1) The pair $\left(\phi^{\rightarrow}, \phi^{\leftarrow}\right)$ is an isotone Galois connection and $\left(\phi^{\leftarrow}(\phi \rightarrow(\lambda)), \phi^{\leftarrow}(\lambda)\right)$ for each $\lambda \in L^{X}$ are attribute oriented concepts with $\phi^{\leftarrow} \in M(Y, X)$.

(2) The pair $\left(\omega_{\phi}, \omega_{\phi}^{\leftarrow}\right)$ is an antitone Galois connection and $\left(\omega_{\phi}^{\leftarrow}\left(\omega_{\phi}(\lambda)\right), \omega_{\phi}(\lambda)\right)$ for $\lambda \in L^{X}$ are formal concepts.

(3) The pair $\left(\xi_{\phi}^{\leftarrow}, \xi_{\phi}\right)$ is an isotone Galois connection and $\left(\xi_{\phi}^{\leftarrow}(\rho), \xi_{\phi}\left(\xi_{\phi}^{\leftarrow}(\rho)\right)\right)$ for each $\rho \in L^{Y}$, are object oriented concepts with $\xi_{\phi}^{\leftarrow}(\rho)=\left(\phi^{\leftarrow}\left(\rho^{*}\right)\right)^{*}$ and $\xi_{\phi}(\lambda)=$ $\left(\phi^{\rightarrow}\left(\lambda^{*}\right)\right)^{*}$. 


\section{The properties of various concepts}

Theorem 2.1. Let $\mathcal{F}(X, Y)=\left\{(\mu, \rho) \in L^{X} \times L^{Y}\right\}$ be a family of formal concepts. For all $\left(\mu_{i}, \rho_{i}\right)_{i \in \Gamma} \in \mathcal{F}(X, Y)$, we define

$$
\begin{aligned}
& \bigvee_{i \in \Gamma}\left(\mu_{i}, \rho_{i}\right)=\left(\omega^{\leftarrow}\left(\bigwedge_{i \in \Gamma} \rho_{i}\right), \bigwedge_{i \in \Gamma} \rho_{i}\right), \\
& \bigwedge_{i \in \Gamma}\left(\mu_{i}, \rho_{i}\right)=\left(\bigwedge_{i \in \Gamma} \mu_{i}, \omega^{\rightarrow}\left(\bigwedge_{i \in \Gamma} \mu_{i}\right)\right) .
\end{aligned}
$$

Then $(\mathcal{F}(X, Y), \vee, \wedge)$ is a complete lattice.

Proof. Since $\bigwedge_{i \in \Gamma} \rho_{i}=\bigwedge_{i \in \Gamma} \omega^{\rightarrow}\left(\mu_{i}\right)=$ $\omega^{\rightarrow}\left(\vee_{i \in \Gamma} \mu_{i}\right)$, by Theorem 1.4(3), $\omega^{\rightarrow}\left(\omega^{\leftarrow}\left(\bigwedge_{i \in \Gamma} \rho_{i}\right)\right)=$ $\omega^{\rightarrow}\left(\omega^{\leftarrow}\left(\omega^{\rightarrow}\left(\bigvee_{i \in \Gamma} \mu_{i}\right)\right)\right)=\omega^{\rightarrow}\left(\vee_{i \in \Gamma} \mu_{i}\right)=$ $\bigwedge_{i \in \Gamma} \rho_{i}$. Hence $\left(\omega^{\leftarrow}\left(\bigwedge_{i \in \Gamma} \rho_{i}\right), \bigwedge_{i \in \Gamma} \rho_{i}\right)=$ $\left(\omega^{\leftarrow}\left(\bigwedge_{i \in \Gamma} \rho_{i}\right), \omega^{\rightarrow}\left(\omega^{\leftarrow}\left(\bigwedge_{i \in \Gamma} \rho_{i}\right)\right)\right) \quad \in \quad \mathcal{F}(X, Y)$. Since $\bigwedge_{i \in \Gamma} \mu_{i}=\bigwedge_{i \in \Gamma} \omega^{\leftarrow}\left(\rho_{i}\right)=\omega^{\leftarrow}\left(\vee_{i \in \Gamma} \rho_{i}\right)$, by Theorem 1.4(3), $\omega^{\leftarrow}\left(\omega \rightarrow\left(\bigwedge_{i \in \Gamma} \mu_{i}\right)\right)=$ $\omega^{\leftarrow}\left(\omega^{\rightarrow}\left(\omega^{\leftarrow}\left(\bigvee_{i \in \Gamma} \rho_{i}\right)\right)\right)=\omega^{\leftarrow}\left(\bigvee_{i \in \Gamma} \rho_{i}\right)=$ $\bigwedge_{i \in \Gamma} \mu_{i}$. Thus, $\left(\bigwedge_{i \in \Gamma} \mu_{i}, \omega \rightarrow\left(\bigwedge_{i \in \Gamma} \mu_{i}\right)\right)=$ $\left(\omega^{\leftarrow}\left(\omega^{\rightarrow}\left(\bigwedge_{i \in \Gamma} \mu_{i}\right)\right), \omega^{\rightarrow}\left(\bigwedge_{i \in \Gamma} \mu_{i}\right)\right) \in \mathcal{F}(X, Y)$.

Theorem 2.2. Let $\mathcal{G}(X, Y)=\left\{(\mu, \rho) \in L^{X} \times L^{Y}\right\}$ be a family of attribute oriented concepts. For all $\left(\mu_{i}, \rho_{i}\right)_{i \in \Gamma} \in$ $\mathcal{G}(X, Y)$, we define

$$
\begin{aligned}
& \bigvee_{i \in \Gamma}\left(\mu_{i}, \rho_{i}\right)=\left(\phi^{\leftarrow}\left(\bigvee_{i \in \Gamma} \rho_{i}\right), \bigvee_{i \in \Gamma} \rho_{i}\right), \\
& \bigwedge_{i \in \Gamma}\left(\mu_{i}, \rho_{i}\right)=\left(\bigwedge_{i \in \Gamma} \mu_{i}, \phi^{\rightarrow}\left(\bigwedge_{i \in \Gamma} \mu_{i}\right)\right) .
\end{aligned}
$$

Then $(\mathcal{G}(X, Y), \vee, \wedge)$ is a complete lattice.

Proof. Since $\bigvee_{i \in \Gamma} \rho_{i}=\bigvee_{i \in \Gamma} \phi \rightarrow\left(\mu_{i}\right)=\phi \rightarrow\left(\bigvee_{i \in \Gamma} \mu_{i}\right)$, by Theorem 1.5(3,4), $\phi^{\rightarrow}\left(\phi^{\leftarrow}\left(\bigvee_{i \in \Gamma} \rho_{i}\right)\right)=$ $\phi^{\rightarrow}\left(\phi^{\leftarrow}\left(\phi^{\rightarrow}\left(\bigvee_{i \in \Gamma} \mu_{i}\right)\right)\right)=\phi^{\rightarrow}\left(\bigvee_{i \in \Gamma} \mu_{i}\right)=$ $\bigvee_{i \in \Gamma} \rho_{i}$. Hence, $\left(\phi^{\leftarrow}\left(\bigvee_{i \in \Gamma} \rho_{i}\right), \bigvee_{i \in \Gamma} \rho_{i}\right)=$ $\left(\phi^{\leftarrow}\left(\bigvee_{i \in \Gamma} \rho_{i}\right), \phi^{\rightarrow}\left(\phi^{\leftarrow}\left(\bigvee_{i \in \Gamma} \rho_{i}\right)\right)\right) \quad \in \quad \mathcal{G}(X, Y)$. Since $\bigwedge_{i \in \Gamma} \mu_{i}=\bigwedge_{i \in \Gamma} \phi^{\leftarrow}\left(\rho_{i}\right)=\phi^{\leftarrow}\left(\bigwedge_{i \in \Gamma} \rho_{i}\right)$, by Theorem 1.5(3,4), $\phi^{\leftarrow}\left(\phi^{\rightarrow}\left(\bigwedge_{i \in \Gamma} \mu_{i}\right)\right)=$ $\phi^{\leftarrow}\left(\phi^{\rightarrow}\left(\phi^{\leftarrow}\left(\wedge_{i \in \Gamma} \rho_{i}\right)\right)\right)=\phi^{\leftarrow}\left(\wedge_{i \in \Gamma} \rho_{i}\right)=$ $\bigwedge_{i \in \Gamma} \mu_{i}$. Hence, $\left.\left(\bigwedge_{i \in \Gamma} \mu_{i}\right), \phi \rightarrow\left(\bigwedge_{i \in \Gamma} \mu_{i}\right)\right)=$ $\left(\phi^{\leftarrow}\left(\phi^{\rightarrow}\left(\bigwedge_{i \in \Gamma} \mu_{i}\right)\right), \phi^{\rightarrow}\left(\bigwedge_{i \in \Gamma} \mu_{i}\right)\right) \in \mathcal{G}(X, Y)$.

Theorem 2.3. Let $\mathcal{H}(X, Y)=\left\{(\mu, \rho) \in L^{X} \times L^{Y}\right\}$ be a family of object oriented concepts. For all $\left(\mu_{i}, \rho_{i}\right)_{i \in \Gamma} \in$ $\mathcal{H}(X, Y)$, we define

$$
\begin{aligned}
& \bigvee_{i \in \Gamma}\left(\mu_{i}, \rho_{i}\right)=\left(\bigvee_{i \in \Gamma} \mu_{i}, \xi^{\rightarrow}\left(\bigvee_{i \in \Gamma} \mu_{i}\right)\right), \\
& \bigwedge_{i \in \Gamma}\left(\mu_{i}, \rho_{i}\right)=\left(\xi^{\leftarrow}\left(\bigwedge_{i \in \Gamma} \rho_{i}\right), \bigwedge_{i \in \Gamma} \rho_{i}\right) .
\end{aligned}
$$

Then $(\mathcal{H}(X, Y), \vee, \wedge)$ is a complete lattice.
Proof. Since $\bigwedge_{i \in \Gamma} \rho_{i}=\bigwedge_{i \in \Gamma} \xi^{\rightarrow}\left(\mu_{i}\right)=$ $\xi \rightarrow\left(\wedge_{i \in \Gamma} \mu_{i}\right)$, by Theorem 1.5(3,4), $\xi^{\rightarrow}\left(\xi^{\leftarrow}\left(\bigwedge_{i \in \Gamma} \rho_{i}\right)\right)=$ $\xi^{\rightarrow}\left(\xi^{\leftarrow}\left(\xi^{\rightarrow}\left(\wedge_{i \in \Gamma} \mu_{i}\right)\right)\right)=\xi^{\rightarrow}\left(\wedge_{i \in \Gamma} \mu_{i}\right)=\bigwedge_{i \in \Gamma} \rho_{i}$. Thus, $\left(\xi^{\leftarrow}\left(\bigwedge_{i \in \Gamma} \rho_{i}\right), \bigwedge_{i \in \Gamma} \rho_{i}\right) \in \mathcal{H}(X, Y)$.

We show $\left(\bigvee_{i \in \Gamma} \mu_{i}, \xi \rightarrow\left(\bigvee_{i \in \Gamma} \mu_{i}\right)\right) \in \mathcal{H}(X, Y)$ from:

Since $\bigvee_{i \in \Gamma} \mu_{i}=\bigvee_{i \in \Gamma} \xi^{\leftarrow}\left(\rho_{i}\right)=\xi^{\leftarrow}\left(\bigvee_{i \in \Gamma} \rho_{i}\right)$, by Theorem 1.5(3,4), $\xi^{\leftarrow}\left(\xi^{\rightarrow}\left(\bigvee_{i \in \Gamma} \mu_{i}\right)\right)=$ $\xi^{\leftarrow}\left(\xi^{\rightarrow}\left(\xi^{\leftarrow}\left(\bigvee_{i \in \Gamma} \rho_{i}\right)\right)\right)=\xi^{\leftarrow}\left(\bigvee_{i \in \Gamma} \rho_{i}\right)=\bigvee_{i \in \Gamma} \mu_{i}$. Hence, $\left.\left(\bigvee_{i \in \Gamma} \mu_{i}\right), \xi \rightarrow\left(\bigvee_{i \in \Gamma} \mu_{i}\right)\right) \in \mathcal{H}(X, Y)$

Definition 2.4. Let $\left(L_{1}, \leq, \vee, \wedge\right)$ and $\left(L_{2}, \leq, \vee, \wedge\right)$ be two lattices. We call:

(1) $L_{1}$ and $L_{2}$ are isomorphic, denoted by $L_{1} \cong L_{2}$, if there exists a bijective function $h: L_{1} \rightarrow L_{2}$ such that $h(x \vee y)=h(x) \vee h(y)$ and $h(x \wedge y)=h(x) \wedge h(y)$.

(2) $L_{1}$ and $L_{2}$ are anti-isomorphic, denoted by $L_{1} \simeq$ $L_{2}$, if there exists a bijective function $g: L_{1} \rightarrow L_{2}$ such that $g(x \vee y)=g(x) \wedge g(y)$ and $g(x \wedge y)=g(x) \vee g(y)$.

Theorem 2.5. Let $\mathcal{G}(X, Y)=\left\{(\mu, \rho) \in L^{X} \times L^{Y}\right\}$ and $\mathcal{H}(X, Y)=\left\{(\mu, \rho) \in L^{X} \times L^{Y}\right\}$ be two families of attribute oriented concepts and object oriented concepts. We define $U: \mathcal{G}(X, Y) \rightarrow \mathcal{H}(X, Y)$ as follows:

$$
U(\mu, \rho)= \begin{cases}\left(\xi_{\phi}^{\leftarrow}\left(\rho^{*}\right), \rho^{*}\right), & \text { if } \mu=\phi^{\leftarrow}(\rho), \\ \left(\mu^{*}, \xi_{\phi}\left(\mu^{*}\right)\right), & \text { if } \rho=\phi^{\rightarrow}(\mu),\end{cases}
$$

where $\xi_{\phi}(\mu)=\left(\phi^{\rightarrow}\left(\mu^{*}\right)\right)^{*}, \xi_{\phi}^{\leftarrow}(\rho)=\left(\phi^{\leftarrow}\left(\rho^{*}\right)\right)^{*}$ and $\left(\phi^{\rightarrow}, \phi^{\leftarrow}\right)$ is an isotone Galois connection. Then $\mathcal{G}(X, Y)$ and $\mathcal{H}(X, Y)$ are anti-isomorphic where $\left(\xi_{\phi}^{\leftarrow}, \xi_{\phi}\right)$ is an isotone Galois connection.

Proof. Since $\xi_{\phi}^{\leftarrow}(\rho) \leq \lambda$ iff $\left(\phi^{\leftarrow}\left(\rho^{*}\right)\right)^{*} \leq \lambda$ iff $\phi^{\leftarrow}\left(\rho^{*}\right) \geq$ $\lambda^{*}$ iff $\phi^{\rightarrow}\left(\lambda^{*}\right) \leq \rho^{*}$ iff $\xi_{\phi}(\lambda) \geq \rho$, for all $\lambda \in L^{X}, \rho \in$ $L^{Y},\left(\xi_{\phi}^{\leftarrow}, \xi_{\phi}\right)$ is an isotone Galois connection. Since $\rho^{*}=$ $\left(\phi^{\rightarrow}(\mu)\right)^{*}=\xi_{\phi}\left(\mu^{*}\right)$ and $\mu^{*}=\left(\phi^{\leftarrow}(\rho)\right)^{*}=\xi_{\phi}^{\leftarrow}\left(\rho^{*}\right)$, then $\left(\xi_{\phi}^{\leftarrow}\left(\rho^{*}\right), \rho^{*}\right)=\left(\xi_{\phi}^{\leftarrow}\left(\xi_{\phi}\left(\mu^{*}\right)\right), \xi_{\phi}\left(\mu^{*}\right)\right) \in \mathcal{H}(X, Y)$ and $\left(\mu^{*}, \xi_{\phi}\left(\mu^{*}\right)\right)=\left(\xi_{\phi}^{\leftarrow}\left(\rho^{*}\right), \xi_{\phi}\left(\xi_{\phi}^{\leftarrow}\left(\rho^{*}\right)\right)\right) \in \mathcal{H}(X, Y)$. Hence $U$ is well defined. For $\left(\xi_{\phi}^{\leftarrow}(\rho), \rho\right) \in \mathcal{H}(X, Y)$, since $\phi^{\rightarrow}\left(\phi^{\leftarrow}\left(\rho^{*}\right)\right)=\phi^{\rightarrow}\left(\left(\xi_{\phi}^{\leftarrow}(\rho)\right)^{*}\right)=\left(\xi_{\phi}\left(\xi_{\phi}^{\leftarrow}(\rho)\right)\right)^{*}=\rho^{*}$, $\left(\phi^{\leftarrow}\left(\rho^{*}\right), \rho^{*}\right)=\left(\phi^{\leftarrow}\left(\rho^{*}\right), \phi^{\rightarrow}\left(\phi^{\leftarrow}\left(\rho^{*}\right)\right)\right) \in \mathcal{G}(X, Y)$ such that $U\left(\phi^{\leftarrow}\left(\rho^{*}\right), \rho^{*}\right)=\left(\xi_{\phi}^{\leftarrow}(\rho), \rho\right)$.

For $\left(\mu, \xi_{\phi}(\mu)\right) \in \mathcal{H}(X, Y)$, since $\phi^{\leftarrow}\left(\phi^{\rightarrow}\left(\mu^{*}\right)\right)=$ $\phi^{\leftarrow}\left(\left(\xi_{\phi}(\mu)\right)^{*}\right)=\left(\xi_{\phi}^{\leftarrow}\left(\left(\xi_{\phi}(\mu)\right)\right)\right)^{*}=\mu^{*}$, $\left(\mu^{*}, \phi^{\rightarrow}\left(\mu^{*}\right)\right) \in \mathcal{G}(X, Y)$ such that $U\left(\mu^{*}, \phi^{\rightarrow}\left(\mu^{*}\right)\right)=$ $\left(\mu, \xi_{\phi}(\mu)\right)$. Hence $U$ is surjective. Trivially, $U$ is injective.

$$
\begin{aligned}
& U\left(\phi^{\leftarrow}\left(\rho_{1}\right), \rho_{1}\right) \wedge U\left(\phi^{\leftarrow}\left(\rho_{2}\right), \rho_{2}\right) \\
& =\left(\xi_{\phi}^{\leftarrow}\left(\rho_{1}^{*}\right), \rho_{1}^{*}\right) \wedge\left(\xi_{\phi}^{\leftarrow}\left(\rho_{2}^{*}\right), \rho_{2}^{*}\right) \\
& \left.=\left(\xi_{\phi}^{\leftarrow}\left(\rho_{1}^{*} \wedge \rho_{2}^{*}\right), \rho_{1}^{*} \wedge \rho_{2}^{*}\right) \text { (by Theorem } 2.3\right) \\
& =\left(\left(\phi^{\leftarrow}\left(\rho_{1} \vee \rho_{2}\right)\right)^{*},\left(\rho_{1} \vee \rho_{2}\right)^{*}\right) \\
& =U\left(\phi^{\leftarrow}\left(\rho_{1} \vee \rho_{2}\right), \rho_{1} \vee \rho_{2}\right) \\
& =U\left(\left(\phi^{\leftarrow}\left(\rho_{1}\right), \rho_{1}\right) \vee\left(\phi^{\leftarrow}\left(\rho_{2}\right), \rho_{2}\right)\right) \text { (by Theorem 2.2) }
\end{aligned}
$$




$$
\begin{aligned}
& U\left(\mu_{1}, \phi \rightarrow\left(\mu_{1}\right)\right) \vee U\left(\mu_{2}, \phi \rightarrow\left(\mu_{2}\right)\right) \\
& =\left(\mu_{1}^{*}, \xi_{\phi}\left(\mu_{1}^{*}\right)\right) \vee\left(\mu_{2}^{*}, \xi_{\phi}\left(\mu_{2}^{*}\right)\right) \\
& =\left(\mu_{1}^{*} \vee \mu_{2}^{*}, \xi_{\phi} \rightarrow\left(\mu_{1}^{*} \vee \mu_{2}^{*}\right)\right) \\
& =U\left(\mu_{1} \wedge \mu_{2}, \phi \rightarrow\left(\mu_{1} \wedge \mu_{2}\right)\right) \\
& =U\left(\left(\mu_{1}, \phi \rightarrow\left(\mu_{1}\right)\right) \wedge\left(\mu_{2}, \phi \rightarrow\left(\mu_{2}\right)\right)\right) \\
& \quad(\text { by Theorem 2.2). }
\end{aligned}
$$

Hence, $\mathcal{G}(X, Y)$ and $\mathcal{H}(X, Y)$ are anti-isomorphic.

Theorem 2.6. Let $\mathcal{F}(X, Y)=\left\{(\mu, \rho) \in L^{X} \times L^{Y}\right\}$ and $\mathcal{G}(X, Y)=\left\{(\mu, \rho) \in L^{X} \times L^{Y}\right\}$ be two families of formal concepts and object oriented concepts. We define $V: \mathcal{F}(X, Y) \rightarrow \mathcal{G}(X, Y)$ as follows:

$$
V(\mu, \rho)= \begin{cases}\left(\phi_{\omega}^{\leftarrow}\left(\rho^{*}\right), \rho^{*}\right), & \text { if } \mu=\omega^{\leftarrow}(\rho), \\ \left(\mu, \phi_{\omega}^{\vec{\omega}}(\mu)\right), & \text { if } \rho=\omega^{\rightarrow}(\mu),\end{cases}
$$

where $\phi_{\omega}(\mu)=\left(\omega^{\rightarrow}(\mu)\right)^{*}, \phi_{\omega}^{\leftarrow}(\rho)=\omega^{\leftarrow}\left(\rho^{*}\right)$ and $\left(\omega^{\rightarrow}, \omega^{\leftarrow}\right)$ is an anti-tone Galois connection. Then $\mathcal{F}(X, Y)$ and $\mathcal{G}(X, Y)$ are isomorphic where $\left(\phi_{\omega}, \phi_{\omega}^{\leftarrow}\right)$ is an isotone Galois connection.

Proof. Since $\phi_{\omega}(\mu) \leq \rho$ iff $\left(\omega^{\rightarrow}(\mu)\right)^{*} \leq \rho$ iff $\omega^{\rightarrow}(\mu) \geq \rho^{*}$ iff $\omega^{\leftarrow}\left(\rho^{*}\right) \geq \mu$ iff $\phi_{\omega}^{\leftarrow}(\rho) \geq \mu$, for all $\mu \in L^{X}, \rho \in L^{Y},\left(\phi_{\omega}, \phi_{\omega}^{\leftarrow}\right)$ is an isotone Galois connection. Since $\phi_{\omega}\left(\phi_{\omega}^{\leftarrow}\left(\rho^{*}\right)\right)=\phi_{\omega}\left(\omega^{\leftarrow}(\rho)\right)=$ $\left(\omega^{\rightarrow}\left(\omega^{\leftarrow}(\rho)\right)\right)^{*}=\rho^{*}$ and $\mu=\omega^{\leftarrow}(\rho)=\phi_{\omega}^{\leftarrow}\left(\rho^{*}\right)$,

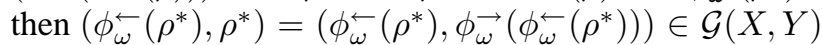
and $\left(\mu, \phi_{\omega}(\mu)\right)=\left(\phi_{\omega}^{\leftarrow}\left(\rho^{*}\right), \phi_{\omega}\left(\phi_{\omega}^{\overleftarrow{\omega}}\left(\rho^{*}\right)\right)\right) \in \mathcal{G}(X, Y)$. Hence $V$ is well defined. For $\left(\phi_{\omega}^{\leftarrow}(\rho), \rho\right) \in \mathcal{G}(X, Y)$, since $\left.\rho=\phi_{\omega}\left(\phi_{\omega}^{\leftarrow}(\rho)\right)\right)=\phi_{\omega}\left(\omega^{\leftarrow}\left(\rho^{*}\right)\right)=\left(\omega^{\rightarrow}\left(\omega^{\leftarrow}\left(\rho^{*}\right)\right)\right)^{*}$, $\left(\omega^{\leftarrow}\left(\rho^{*}\right), \rho^{*}\right)=\left(\omega^{\leftarrow}\left(\rho^{*}\right), \omega^{\rightarrow}\left(\omega^{\leftarrow}\left(\rho^{*}\right)\right)\right) \in \mathcal{F}(X, Y)$ such that $V\left(\omega^{\leftarrow}\left(\rho^{*}\right), \rho^{*}\right)=\left(\phi_{\omega}^{\leftarrow}(\rho), \rho\right)$.

For $\left(\mu, \phi_{\omega}(\mu)\right) \in \mathcal{G}(X, Y)$, since $\mu=$ $\phi_{\omega}^{\leftarrow}\left(\phi_{\omega}(\mu)\right)=\phi_{\omega}^{\leftarrow}\left((\omega \rightarrow(\mu))^{*}\right)=\omega^{\leftarrow}(\omega \rightarrow(\mu))$ $(\mu, \omega \rightarrow(\mu))=\left(\omega^{\leftarrow}(\omega \rightarrow(\mu)), \omega^{\rightarrow}(\mu)\right) \in \mathcal{F}(X, Y)$ such that $V(\mu, \omega \rightarrow(\mu))=\left(\mu, \phi_{\omega}(\mu)\right)$. Hence $V$ is surjective. Trivially, $V$ is injective.

$$
\begin{aligned}
& V\left(\omega^{\leftarrow}\left(\rho_{1}\right), \rho_{1}\right) \vee V\left(\omega^{\leftarrow}\left(\rho_{2}\right), \rho_{2}\right) \\
& =\left(\phi_{\omega}^{\leftarrow}\left(\rho_{1}^{*}\right), \rho_{1}^{*}\right) \vee\left(\phi_{\omega}^{\leftarrow}\left(\rho_{2}^{*}\right), \rho_{2}^{*}\right) \\
& =\left(\phi_{\omega}^{\leftarrow}\left(\rho_{1}^{*} \vee \rho_{2}^{*}\right), \rho_{1}^{*} \vee \rho_{2}^{*}\right) \\
& =V\left(\omega^{\leftarrow}\left(\rho_{1} \wedge \rho_{2}\right), \rho_{1} \wedge \rho_{2}\right) \\
& \left.=V\left(\omega^{\leftarrow}\left(\rho_{1}\right), \rho_{1}\right) \vee\left(\omega^{\leftarrow}\left(\rho_{2}\right), \rho_{2}\right)\right) . \\
& V\left(\mu_{1}, \omega \rightarrow\left(\mu_{1}\right)\right) \wedge V\left(\mu_{2}, \omega \rightarrow\left(\mu_{2}\right)\right) \\
& =\left(\mu_{1}, \phi_{\omega}\left(\mu_{1}\right)\right) \wedge\left(\mu_{2}, \phi_{\omega}\left(\mu_{2}\right)\right) \\
& =\left(\mu_{1} \wedge \mu_{2}, \phi_{\omega}\left(\mu_{1} \wedge \mu_{2}\right)\right) \\
& =V\left(\mu_{1} \wedge \mu_{2}, \omega \rightarrow\left(\mu_{1} \wedge \mu_{2}\right)\right) \\
& =V\left(\left(\mu_{1}, \omega^{\rightarrow}\left(\mu_{1}\right)\right) \wedge\left(\mu_{2}, \omega \rightarrow\left(\mu_{2}\right)\right)\right)
\end{aligned}
$$

Hence, $\mathcal{F}(X, Y)$ and $\mathcal{G}(X, Y)$ are isomorphic.
Theorem 2.7. Let $\mathcal{F}(X, Y)=\left\{(\mu, \rho) \in L^{X} \times L^{Y}\right\}$ and $\mathcal{H}(X, Y)=\left\{(\mu, \rho) \in L^{X} \times L^{Y}\right\}$ be two families of formal concepts and attribute oriented concepts. We define $W: \mathcal{F}(X, Y) \rightarrow \mathcal{H}(X, Y)$ as follows:

$$
W(\mu, \rho)= \begin{cases}\left(\xi_{\omega}^{\leftarrow}(\rho), \rho\right), & \text { if } \mu=\omega^{\leftarrow}(\rho), \\ \left(\mu^{*}, \xi_{\omega}\left(\mu^{*}\right)\right), & \text { if } \rho=\omega^{\rightarrow}(\mu),\end{cases}
$$

where $\xi_{\omega}(\mu)=\omega^{\rightarrow}\left(\mu^{*}\right), \quad \xi_{\omega}^{\leftarrow}(\rho)=\left(\omega^{\leftarrow}(\rho)\right)^{*}$ and $\left(\omega^{\rightarrow}, \omega^{\leftarrow}\right)$ is an anti-tone Galois connection. Then $\mathcal{F}(X, Y)$ and $\mathcal{H}(X, Y)$ are anti-isomorphic where $\left(\xi_{\omega}^{\leftarrow}, \xi_{\omega}\right)$ is an isotone Galois connection.

Proof. Since $\xi_{\omega}^{\leftarrow}(\rho) \leq \lambda$ iff $\left(\omega^{\leftarrow}(\rho)\right)^{*} \leq \lambda$ iff $\omega^{\leftarrow}(\rho) \geq$ $\lambda^{*}$ iff $\omega \rightarrow\left(\lambda^{*}\right) \geq \rho$ iff $\xi_{\omega}(\lambda) \geq \rho$, for all $\lambda \in L^{X}, \rho \in L^{Y}$, $\left(\xi_{\omega}^{\leftarrow}, \xi_{\omega}\right)$ is an isotone Galois connection.

Since $\rho=\omega^{\rightarrow}(\mu)=\xi_{\omega}\left(\mu^{*}\right)$ and $\mu=\omega^{\leftarrow}(\rho)=$ $\left(\xi_{\omega}^{\leftarrow}(\rho)\right)^{*}$, then $\left(\xi_{\omega}^{\leftarrow}(\rho), \rho\right)=\left(\xi_{\omega}^{\leftarrow}\left(\xi_{\omega}\left(\mu^{*}\right)\right), \xi_{\omega}\left(\mu^{*}\right)\right) \in$ $\mathcal{H}(X, Y)$ and $\left(\mu^{*}, \xi_{\omega}\left(\mu^{*}\right)\right)=\left(\xi_{\omega}^{\leftarrow}(\rho), \xi_{\omega}\left(\xi_{\omega}^{\leftarrow}(\rho)\right)\right) \in$ $\mathcal{H}(X, Y)$. Hence $W$ is well defined. For $\left(\xi_{\omega}^{\leftarrow}(\rho), \rho\right) \in$ $\mathcal{H}(X, Y)$, there exists $\left(\omega^{\leftarrow}(\rho), \rho\right) \in \mathcal{F}(X, Y)$ such that $W(\omega \leftarrow(\rho), \rho)=\left(\xi_{\omega}^{\leftarrow}(\rho), \rho\right)$

For $\left(\mu, \xi_{\omega}(\mu)\right) \in \mathcal{H}(X, Y)$, since $\mu=\xi_{\omega}^{\leftarrow}\left(\xi_{\omega}(\mu)\right)=$ $\xi_{\omega}^{\leftarrow}\left(\omega^{\rightarrow}\left(\mu^{*}\right)\right)=\left(\omega^{\leftarrow}\left(\omega^{\rightarrow}\left(\mu^{*}\right)\right)\right)^{*}, \quad\left(\mu^{*}, \omega^{\rightarrow}\left(\mu^{*}\right)\right)=$ $\left(\omega^{\leftarrow}\left(\omega^{\rightarrow}\left(\mu^{*}\right)\right), \omega^{\rightarrow}\left(\mu^{*}\right)\right) \in \mathcal{F}(X, Y)$ such that $W\left(\mu^{*}, \omega \rightarrow\left(\mu^{*}\right)\right)=\left(\mu, \xi_{\omega}(\mu)\right)$. Hence $W$ is surjective. Trivially, $W$ is injective.

$$
\begin{aligned}
& W\left(\omega^{\leftarrow}\left(\rho_{1}\right), \rho_{1}\right) \wedge W\left(\omega^{\leftarrow}\left(\rho_{2}\right), \rho_{2}\right) \\
& =\left(\left(\omega^{\leftarrow}\left(\rho_{1}\right)\right)^{*}, \rho_{1}\right) \wedge\left(\left(\omega^{\leftarrow}\left(\rho_{2}\right)\right)^{*}, \rho_{2}\right) \\
& =\left(\xi_{\omega}^{\leftarrow}\left(\rho_{1}\right), \rho_{1}\right) \wedge\left(\xi_{\omega}^{\leftarrow}\left(\rho_{2}\right), \rho_{2}\right) \\
& =\left(\xi_{\omega}^{\leftarrow}\left(\rho_{1} \wedge \rho_{2}\right), \rho_{1} \wedge \rho_{2}\right) \\
& =W\left(\omega^{\leftarrow}\left(\rho_{1} \wedge \rho_{2}\right), \rho_{1} \wedge \rho_{2}\right) \\
& =W\left(\left(\omega^{\leftarrow}\left(\rho_{1}\right), \rho_{1}\right) \vee\left(\omega^{\leftarrow}\left(\rho_{2}\right), \rho_{2}\right)\right) . \\
& W\left(\mu_{1}, \omega^{\rightarrow}\left(\mu_{1}\right)\right) \vee W\left(\mu_{2}, \omega \rightarrow\left(\mu_{2}\right)\right) \\
& =\left(\mu_{1}^{*}, \xi_{\omega}\left(\mu_{1}^{*}\right)\right) \vee\left(\mu_{2}^{*}, \xi_{\omega}\left(\mu_{2}^{*}\right)\right) \\
& =\left(\mu_{1}^{*} \vee \mu_{2}^{*}, \xi_{\omega}\left(\mu_{1}^{*} \vee \mu_{2}^{*}\right)\right) \\
& =W\left(\mu_{1} \wedge \mu_{2}, \omega^{\rightarrow}\left(\mu_{1} \wedge \mu_{2}\right)\right) \\
& =W\left(\left(\mu_{1}, \omega^{\rightarrow}\left(\mu_{1}\right)\right) \wedge\left(\mu_{2}, \omega \rightarrow\left(\mu_{2}\right)\right)\right) .
\end{aligned}
$$

Then $\mathcal{F}(X, Y)$ and $\mathcal{H}(X, Y)$ are anti-isomorphic.

Definition 2.8. Let $\mathcal{F}(X, Y)=\left\{(\mu, \rho) \in L^{X} \times L^{Y}\right\}$ (resp. $\mathcal{G}(X, Y)$ or $\mathcal{H}(X, Y)$ ) be a family of formal concepts(resp. attribute oriented concepts or object oriented concepts). Then:

(1) attribute $a \in Y$ is said to be dispensable if $\mathcal{F}(X, Y) \cong \mathcal{F}(X, Y-\{a\})($ resp. $\mathcal{G}(X, Y) \cong \mathcal{G}(X, Y-$ $\{a\})$ or $\mathcal{H}(X, Y) \cong \mathcal{H}(X, Y-\{a\}))$, otherwise attribute $a \in Y$ is said to be indispensable.

(2) attributes set $B \subset Y$ is said to be a consistent set if $\mathcal{F}(X, Y) \cong \mathcal{F}(X, B)$ (resp. $\mathcal{G}(X, Y) \cong \mathcal{G}(X, B)$ or $\mathcal{H}(X, Y) \cong \mathcal{H}(X, B))$. Furthermore, B is called otherwise attribute $a \in Y$ is said to be indispensable. 
(3) $B \subset Y$ is called a reduct if $B$ is a consistent set and $\mathcal{F}(X, Y) \nVdash \mathcal{F}(X, B-\{b\})$ (resp. $\mathcal{G}(X, Y) \not$ $\mathcal{G}(X, B-\{b\})$ or $\mathcal{H}(X, Y) \neq \mathcal{H}(X, B-\{b\}))$ for each $b \in B$.

Example 2.9. Let $X=\{a, b\}$ and $Y=\{u, v, w\}$ be sets. Let $\left(L=\left\{0, \frac{1}{2}, 1\right\}, \odot\right)$ be a complete residuated lattice such that $x \odot y=(x+y-1) \vee 1, x \rightarrow y=(1-x+y) \wedge 1$, $x^{*}=1-x$. For $\lambda \in L^{X}$, we denote $(\lambda, \omega \rightarrow(\lambda))=$ $\left((\lambda(a), \lambda(b)),\left(\omega^{\rightarrow}(\lambda)(u), \omega \rightarrow(\lambda)(v), \omega^{\rightarrow}(\lambda)(w)\right)\right.$, we define;

$((0,0),(1,1,1)) \quad\left(\left(0, \frac{1}{2}\right),\left(\frac{1}{2}, 1,1\right)\right) \quad\left((0,1),\left(0,1, \frac{1}{2}\right)\right)$ $\left(\left(\frac{1}{2}, 0\right),\left(\frac{1}{2}, 1, \frac{1}{2}\right)\right) \quad\left(\left(\frac{1}{2}, \frac{1}{2}\right),\left(\frac{1}{2}, 1, \frac{1}{2}\right)\right) \quad\left(\left(\frac{1}{2}, 1\right),\left(0,1, \frac{1}{2}\right)\right)$ $\left((1,0),\left(0, \frac{1}{2}, 0\right)\right) \quad\left(\left(1, \frac{1}{2}\right),\left(0, \frac{1}{2}, 0\right)\right) \quad\left((1,1),\left(0, \frac{1}{2}, 0\right)\right)$

Then $\omega \rightarrow \in K(X, Y)$. By Theorem 1.7, we obtain

$$
\omega^{\leftarrow}(\rho)=\bigvee\left\{\lambda \in L^{X} \mid \omega^{\rightarrow}(\lambda) \geq \rho\right\}
$$

For $\rho \in L^{Y}$, we denote $\left(\rho, \omega^{\leftarrow}(\rho)\right)=$ $\left((\rho(u), \rho(v), \rho(w)),\left(\omega^{\leftarrow}(\rho)(a), \omega^{\leftarrow}(\rho)(b)\right)\right)$,

$$
\begin{array}{lll}
((1,1,1),(0,0)) & \left(\left(1,1, \frac{1}{2}\right),(0,0)\right) & ((1,1,0),(0,0)) \\
\left(\left(1, \frac{1}{2}, 1\right),(0,0)\right) & \left(\left(1, \frac{1}{2}, \frac{1}{2}\right),(0,0)\right) & \left(\left(1, \frac{1}{2}, 0\right),(0,0)\right) \\
((1,0,1),(0,0)) & \left(\left(1,0, \frac{1}{2}\right),(0,0)\right) & ((1,0,0),(0,0)) \\
\left(\left(\frac{1}{2}, 1,1\right),\left(0, \frac{1}{2}\right)\right) & \left(\left(\frac{1}{2}, 1, \frac{1}{2}\right),\left(\frac{1}{2}, \frac{1}{2}\right)\right) & \left(\left(\frac{1}{2}, 1,0\right),\left(\frac{1}{2}, \frac{1}{2}\right)\right) \\
\left(\left(\frac{1}{2}, \frac{1}{2}, 1\right),\left(0, \frac{1}{2}\right)\right) & \left(\left(\frac{1}{2}, \frac{1}{2}, \frac{1}{2}\right),\left(\frac{1}{2}, \frac{1}{2}\right)\right) & \left(\left(\frac{1}{2}, \frac{1}{2}, 0\right),\left(\frac{1}{2}, \frac{1}{2}\right)\right) \\
\left(\left(\frac{1}{2}, 0,1\right),\left(0, \frac{1}{2}\right)\right) & \left(\left(\frac{1}{2}, 0, \frac{1}{2}\right),\left(\frac{1}{2}, \frac{1}{2}\right)\right) & \left(\left(\frac{1}{2}, 0,0\right),\left(\frac{1}{2}, \frac{1}{2}\right)\right) \\
\left((0,1,1),\left(0, \frac{1}{2}\right)\right) & \left(\left(0,1, \frac{1}{2}\right),\left(\frac{1}{2}, 1\right)\right) & \left((0,1,0),\left(\frac{1}{2}, \frac{1}{2}\right)\right) \\
\left(\left(0, \frac{1}{2}, 1\right),\left(0, \frac{1}{2}\right)\right) & \left(\left(0, \frac{1}{2}, \frac{1}{2}\right),\left(\frac{1}{2}, 1\right)\right) & \left(\left(0, \frac{1}{2}, 0\right),(1,1)\right) \\
\left((0,0,1),\left(0, \frac{1}{2}\right)\right) & \left(\left(0,0, \frac{1}{2}\right),\left(\frac{1}{2}, 1\right)\right) & ((0,0,0),(1,1))
\end{array}
$$

We obtain a formal concept family as follows:

$$
\begin{aligned}
& \mathcal{F}(X, Y)=\left\{((0,0),(1,1,1)),\left(\left(0, \frac{1}{2}\right),\left(\frac{1}{2}, 1,1\right)\right),\right. \\
& \left.\left(\left(\frac{1}{2}, \frac{1}{2}\right),\left(\frac{1}{2}, 1, \frac{1}{2}\right)\right),\left(\left(\frac{1}{2}, 1\right),\left(0,1, \frac{1}{2}\right)\right),\left((1,1),\left(0, \frac{1}{2}, 0\right)\right)\right\}
\end{aligned}
$$

$v \in Y$ is dispensable for $\mathcal{F}(X, Y)$ because $\mathcal{F}(X, Y) \cong$ $\mathcal{F}(X, Y-\{v\}) . \quad B=\{u, w\}$ is a consistent set for $\mathcal{F}(X, Y)$ because $\mathcal{F}(X, Y) \cong \mathcal{F}(X, B)$ where

$$
\begin{aligned}
& \mathcal{F}(X, B)=\left\{((0,0),(1,1)),\left(\left(0, \frac{1}{2}\right),\left(\frac{1}{2}, 1\right)\right),\right. \\
& \left.\left(\left(\frac{1}{2}, \frac{1}{2}\right),\left(\frac{1}{2}, \frac{1}{2}\right)\right),\left(\left(\frac{1}{2}, 1\right),\left(0, \frac{1}{2}\right)\right),((1,1),(0,0))\right\}
\end{aligned}
$$

Moreover, $B$ is a reduct for $\mathcal{F}(X, Y)$ because $B$ is a consistent set and $\mathcal{F}(X, Y) \not \mathcal{F}(X, B-\{u\})$ and $\mathcal{F}(X, Y) \not$ $\mathcal{F}(X, B-\{w\})$.

For $\lambda \in L^{X}$, we denote $\left(\lambda, \phi_{\omega}(\lambda)\right)=$ $\left((\lambda(a), \lambda(b)),\left(\phi_{\omega}(\lambda)(u), \phi_{\omega}(\lambda)(v), \phi_{\omega}(\lambda)(w)\right)\right.$, we obtain;

$$
\begin{array}{ccc}
((0,0),(0,0,0)) & \left(\left(0, \frac{1}{2}\right),\left(\frac{1}{2}, 0,0\right)\right) & \left((0,1),\left(1,0, \frac{1}{2}\right)\right) \\
\left(\left(\frac{1}{2}, 0\right),\left(\frac{1}{2}, 0, \frac{1}{2}\right)\right) & \left(\left(\frac{1}{2}, \frac{1}{2}\right),\left(\frac{1}{2}, 0, \frac{1}{2}\right)\right. & \left(\left(\frac{1}{2}, 1\right),\left(1,0, \frac{1}{2}\right)\right) \\
\left((1,0),\left(1, \frac{1}{2}, 1\right)\right) & \left(\left(1, \frac{1}{2}\right),\left(1, \frac{1}{2}, 1\right)\right) & \left((1,1),\left(1, \frac{1}{2}, 1\right)\right)
\end{array}
$$

For $\rho \in L^{Y}$, we denote $\left(\rho, \phi_{\omega}^{\leftarrow}(\rho)\right)=$ $\left((\rho(u), \rho(v), \rho(w)),\left(\phi_{\omega}^{\leftarrow}(\rho)(a), \phi_{\omega}^{\leftarrow}(\rho)(b)\right)\right)$,

$\begin{array}{lll}((0,0,0),(0,0)) & \left(\left(0,0, \frac{1}{2}\right),(0,0)\right) & ((0,0,1),(0,0)) \\ \left(\left(0, \frac{1}{2}, 0\right),(0,0)\right) & \left(\left(0, \frac{1}{2}, \frac{1}{2}\right),(0,0)\right) & \left(\left(0, \frac{1}{2}, \frac{1}{2}\right),(0,0)\right) \\ ((0,1,0),(0,0)) & \left(\left(0,1, \frac{1}{2}\right),(0,0)\right) & ((0,1,1),(0,0)) \\ \left(\left(\frac{1}{2}, 0,0\right),\left(0, \frac{1}{2}\right)\right) & \left(\left(\frac{1}{2}, 0, \frac{1}{2}\right),\left(\frac{1}{2}, \frac{1}{2}\right)\right) & \left(\left(\frac{1}{2}, 0,1\right),\left(\frac{1}{2}, \frac{1}{2}\right)\right) \\ \left(\left(\frac{1}{2}, \frac{1}{2}, 0\right),\left(0, \frac{1}{2}\right)\right) & \left(\left(\frac{1}{2}, \frac{1}{2}, \frac{1}{2}\right),\left(\frac{1}{2}, \frac{1}{2}\right)\right) & \left(\left(\frac{1}{2}, \frac{1}{2}, 1\right),\left(\frac{1}{2}, \frac{1}{2}\right)\right) \\ \left(\left(\frac{1}{2}, 1,0\right),\left(0, \frac{1}{2}\right)\right) & \left(\left(\frac{1}{2}, 1, \frac{1}{2}\right),\left(\frac{1}{2}, \frac{1}{2}\right)\right) & \left(\left(\frac{1}{2}, 1,1\right),\left(\frac{1}{2}, \frac{1}{2}\right)\right) \\ \left((1,0,0),\left(0, \frac{1}{2}\right)\right) & \left(\left(1,0, \frac{1}{2}\right),\left(\frac{1}{2}, 1\right)\right) & \left((1,0,1),\left(\frac{1}{2}, \frac{1}{2}\right)\right) \\ \left(\left(1, \frac{1}{2}, 0\right),\left(0, \frac{1}{2}\right)\right) & \left(\left(1, \frac{1}{2}, \frac{1}{2}\right),\left(\frac{1}{2}, 1\right)\right) & \left(\left(1, \frac{1}{2}, 1\right),(1,1)\right) \\ \left((1,1,0),\left(0, \frac{1}{2}\right)\right) & \left(\left(1,1, \frac{1}{2}\right),\left(\frac{1}{2}, 1\right)\right) & ((1,1,1),(1,1))\end{array}$

We obtain an attribute oriented concept family as follows:

$\mathcal{G}(X, Y)=\left\{((0,0),(0,0,0)),\left(\left(0, \frac{1}{2}\right),\left(\frac{1}{2}, 0,0\right)\right)\right.$, $\left.\left(\left(\frac{1}{2}, \frac{1}{2}\right),\left(\frac{1}{2}, 0, \frac{1}{2}\right)\right),\left(\left(\frac{1}{2}, 1\right),\left(1,0, \frac{1}{2}\right)\right),\left((1,1),\left(1, \frac{1}{2}, 1\right)\right)\right\}$

$v \in Y$ is dispensable for $\mathcal{G}(X, Y)$ because $\mathcal{G}(X, Y) \cong$ $\mathcal{G}(X, Y-\{v\}) . B=\{u, w\}$ is a consistent set for $\mathcal{G}(X, Y)$ because $\mathcal{G}(X, Y) \cong \mathcal{G}(X, B)$ where

$$
\begin{gathered}
\mathcal{G}(X, B)=\left\{((0,0),(0,0)),\left(\left(0, \frac{1}{2}\right),\left(\frac{1}{2}, 0\right)\right),\right. \\
\left.\left(\left(\frac{1}{2}, \frac{1}{2}\right),\left(\frac{1}{2}, \frac{1}{2}\right)\right),\left(\left(\frac{1}{2}, 1\right),\left(1, \frac{1}{2}\right)\right),((1,1),(1,1))\right\}
\end{gathered}
$$

Moreover, $B$ is a reduct for $\mathcal{G}(X, Y)$ because $B$ is a consistent set and $\mathcal{G}(X, Y) \not \mathcal{G}(X, B-\{u\})$ and $\mathcal{G}(X, Y) \not$ $\mathcal{G}(X, B-\{w\})$.

We define $V: \mathcal{F}(X, Y) \rightarrow \mathcal{G}(X, Y)$ as follows:

$$
\begin{aligned}
& V((0,0),(1,1,1))=((0,0),(0,0,0)) \\
& V\left(\left(0, \frac{1}{2}\right),\left(\frac{1}{2}, 1,1\right)\right)=\left(\left(0, \frac{1}{2}\right),\left(\frac{1}{2}, 0,0\right)\right) \\
& V\left(\left(\frac{1}{2}, \frac{1}{2}\right),\left(\frac{1}{2}, 1, \frac{1}{2}\right)\right)=\left(\left(\frac{1}{2}, \frac{1}{2}\right),\left(\frac{1}{2}, 0, \frac{1}{2}\right)\right) \\
& V\left(\left(\frac{1}{2}, 1\right),\left(0,1, \frac{1}{2}\right)\right)=\left(\left(\frac{1}{2}, 1\right),\left(1,0, \frac{1}{2}\right)\right) \\
& V\left((1,1),\left(0, \frac{1}{2}, 0\right)\right)=\left((1,1),\left(1, \frac{1}{2}, 1\right)\right) .
\end{aligned}
$$

Then $\mathcal{F}(X, Y) \cong \mathcal{G}(X, Y)$.

For $\rho \in L^{Y}$, we denote $\left(\rho, \xi_{\omega}^{\leftarrow}(\rho)\right)=$ $\left((\rho(u), \rho(v), \rho(w)),\left(\xi_{\omega}^{\leftarrow}(\rho)(a), \xi_{\omega}^{\leftarrow}(\rho)(b)\right)\right)$

$\begin{array}{ccc}((0,0,0),(0,0)) & \left(\left(0,0, \frac{1}{2}\right),\left(\frac{1}{2}, 0\right)\right) & \left((0,0,1),\left(1, \frac{1}{2}\right)\right) \\ \left(\left(0, \frac{1}{2}, 0\right),(0,0)\right) & \left(\left(0, \frac{1}{2}, \frac{1}{2}\right),\left(\frac{1}{2}, 0\right)\right) & \left(\left(0, \frac{1}{2}, 1\right),\left(1, \frac{1}{2}\right)\right) \\ \left((0,1,0),\left(\frac{1}{2}, 0\right)\right) & \left(\left(0,1, \frac{1}{2}\right),\left(\frac{1}{2}, 0\right)\right) & \left((0,1,1),\left(1, \frac{1}{2}\right)\right) \\ \left(\left(\frac{1}{2}, 0,0\right),\left(\frac{1}{2}, \frac{1}{2}\right)\right) & \left(\left(\frac{1}{2}, 0, \frac{1}{2}\right),\left(\frac{1}{2}, \frac{1}{2}\right)\right) & \left(\left(\frac{1}{2}, 0,1\right),\left(1, \frac{1}{2}\right)\right) \\ \left(\left(\frac{1}{2}, \frac{1}{2}, 0\right),\left(\frac{1}{2}, \frac{1}{2}\right)\right) & \left(\left(\frac{1}{2}, \frac{1}{2}, \frac{1}{2}\right),\left(\frac{1}{2}, \frac{1}{2}\right)\right) & \left(\left(\frac{1}{2}, \frac{1}{2}, 1\right),\left(1, \frac{1}{2}\right)\right) \\ \left(\left(\frac{1}{2}, 1,0\right),\left(\frac{1}{2}, \frac{1}{2}\right)\right) & \left(\left(\frac{1}{2}, 1, \frac{1}{2}\right),\left(\frac{1}{2}, \frac{1}{2}\right)\right) & \left(\left(\frac{1}{2}, 1,1\right),\left(1, \frac{1}{2}\right)\right) \\ ((1,0,0),(1,1)) & \left(\left(1,0, \frac{1}{2}\right),(1,1)\right) & ((1,0,1),(1,1)) \\ \left(\left(1, \frac{1}{2}, 0\right),(1,1)\right) & \left(\left(1, \frac{1}{2}, \frac{1}{2}\right),(1,1)\right) & \left(\left(1, \frac{1}{2}, 1\right),(1,1)\right) \\ ((1,1,0),(1,1)) & \left(\left(1,1, \frac{1}{2}\right),(1,1)\right) & ((1,1,1),(1,1))\end{array}$

For $\lambda \in L^{X}$, we denote $\left(\lambda, \xi_{\omega}(\lambda)\right)=$ $\left((\lambda(a), \lambda(b)),\left(\xi_{\omega}(\lambda)(u), \xi_{\omega}(\lambda)(v), \xi_{\omega}(\lambda)(w)\right)\right.$, we obtain;

$$
\begin{array}{ccc}
\left((0,0),\left(0, \frac{1}{2}, 0\right)\right) & \left(\left(0, \frac{1}{2}\right),\left(0, \frac{1}{2}, 0\right)\right) & \left((0,1),\left(0, \frac{1}{2}, 0\right)\right) \\
\left(\left(\frac{1}{2}, 0\right),\left(0,1, \frac{1}{2}\right)\right) & \left(\left(\frac{1}{2}, \frac{1}{2}\right),\left(\frac{1}{2}, 1, \frac{1}{2}\right)\right) & \left(\left(\frac{1}{2}, 1\right),\left(\frac{1}{2}, 1, \frac{1}{2}\right)\right) \\
\left((1,0),\left(0,1, \frac{1}{2}\right)\right) & \left(\left(1, \frac{1}{2}\right),\left(\frac{1}{2}, 1,1\right)\right) & ((1,1),(1,1,1))
\end{array}
$$


We obtain an object oriented concept family as follows:

$$
\begin{gathered}
\mathcal{H}(X, Y)=\left\{\left((0,0),\left(0, \frac{1}{2}, 0\right)\right),\left(\left(\frac{1}{2}, 0\right),\left(0,1, \frac{1}{2}\right)\right),\right. \\
\left.\left(\left(\frac{1}{2}, \frac{1}{2}\right),\left(\frac{1}{2}, 1, \frac{1}{2}\right)\right),\left(\left(1, \frac{1}{2}\right),\left(\frac{1}{2}, 1,1\right)\right),((1,1),(1,1,1))\right\}
\end{gathered}
$$

$v \in Y$ is dispensable for $\mathcal{H}(X, Y)$ because $\mathcal{H}(X, Y) \cong$ $\mathcal{H}(X, Y-\{v\}) . \quad B=\{u, w\}$ is a consistent set for $\mathcal{H}(X, Y)$ because $\mathcal{H}(X, Y) \cong \mathcal{H}(X, B)$ where

$$
\begin{gathered}
\mathcal{H}(X, B)=\left\{((0,0),(0,0)),\left(\left(\frac{1}{2}, 0\right),\left(0, \frac{1}{2}\right)\right),\right. \\
\left.\left(\left(\frac{1}{2}, \frac{1}{2}\right),\left(\frac{1}{2}, \frac{1}{2}\right)\right),\left(\left(1, \frac{1}{2}\right),\left(\frac{1}{2}, 1\right)\right),((1,1),(1,1))\right\}
\end{gathered}
$$

Moreover, $B$ is a reduct for $\mathcal{H}(X, Y)$ because $B$ is a consistent set and $\mathcal{H}(X, Y) \neq \mathcal{H}(X, B-\{u\})$ and $\mathcal{H}(X, Y) \neq$ $\mathcal{H}(X, B-\{w\})$.

We define $W: \mathcal{F}(X, Y) \rightarrow \mathcal{H}(X, Y)$ as follows:

$$
\begin{aligned}
& W((0,0),(1,1,1))=((1,1),(1,1,1)) \\
& W\left(\left(0, \frac{1}{2}\right),\left(\frac{1}{2}, 1,1\right)\right)=\left(\left(1, \frac{1}{2}\right),\left(\frac{1}{2}, 1,1\right)\right) \\
& W\left(\left(\frac{1}{2}, \frac{1}{2}\right),\left(\frac{1}{2}, 1, \frac{1}{2}\right)\right)=\left(\left(\frac{1}{2}, \frac{1}{2}\right),\left(\frac{1}{2}, 1, \frac{1}{2}\right)\right) \\
& W\left(\left(\frac{1}{2}, 1\right),\left(0,1, \frac{1}{2}\right)\right)=\left(\left(\frac{1}{2}, 0\right),\left(0,1, \frac{1}{2}\right)\right) \\
& W\left((1,1),\left(0, \frac{1}{2}, 0\right)\right)=\left((1,1),\left(0, \frac{1}{2}, 0\right)\right) .
\end{aligned}
$$

Then $\mathcal{F}(X, Y) \cong \mathcal{H}(X, Y)$.

We define $U: \mathcal{G}(X, Y) \rightarrow \mathcal{H}(X, Y)$ as follows:

$$
\begin{aligned}
& U((0,0),(0,0,0))=((1,1),(1,1,1)) \\
& U\left(\left(0, \frac{1}{2}\right),\left(\frac{1}{2}, 0,0\right)\right)=\left(\left(1, \frac{1}{2}\right),\left(\frac{1}{2}, 1,1\right)\right) \\
& U\left(\left(\frac{1}{2}, \frac{1}{2}\right),\left(\frac{1}{2}, 0, \frac{1}{2}\right)\right)=\left(\left(\frac{1}{2}, \frac{1}{2}\right),\left(\frac{1}{2}, 1, \frac{1}{2}\right)\right) \\
& U\left(\left(\frac{1}{2}, 1\right),\left(1,0, \frac{1}{2}\right)\right)=\left(\left(\frac{1}{2}, 0\right),\left(0,1, \frac{1}{2}\right)\right) \\
& U\left((1,1),\left(1, \frac{1}{2}, 1\right)\right)=\left((1,1),\left(0, \frac{1}{2}, 0\right)\right) .
\end{aligned}
$$

Then $\mathcal{G}(X, Y) \simeq \mathcal{H}(X, Y)$.

\section{References}

[1] R. Bělohlávek, "Similarity relations in concept lattices," J. Logic and Computation, vol. 10, no 6,pp.823-845, 2000.

[2] R. Bělohlávek, "Lattices of fixed points of Galois connections," Math. Logic Quart., vol. 47, pp.111-116, 2001.

[3] R. Bělohlávek, "Concept lattices and order in fuzzy logic," Ann. Pure Appl. Logic, vol. 128, pp.277-298, 2004.
[4] R. Bělohlávek, Fuzzy relational systems, Kluwer Academic Publisher, New York, 2002.

[5] G. Georgescu, A. Popescue," Non-dual fuzzy connections," Arch. Math. Log., vol. 43, pp. 1009-1039, 2004.

[6] U. Höhle, E. P. Klement, Non-classical logic and their applications to fuzzy subsets, Kluwer Academic Publisher, Boston, 1995.

[7] H. Lai, D. Zhang," Concept lattices of fuzzy contexts: Formal concept analysis vs. rough set theory," Int. J. Approx. Reasoning, vol. 50, pp.695-707, 2009.

[8] Y.C. Kim, J.W. Park, "Join preserving maps and various concepts," Int.J. Contemp. Math. Sciences, vol. 5, no.5, pp.243-251, 2010.

[9] J.M. Ko, Y.C. Kim, ”Antitone Galois connections and formal concepts," Int. J. Fuzzy Logic and Intelligent Systems, vol.10, no.2, pp.107-112, 2010.

[10] E. Turunen, Mathematics Behind Fuzzy Logic, A Springer-Verlag Co., 1999.

[11] R. Wille, Restructuring lattice theory; an approach based on hierarchies of concept, in: 1. Rival(Ed.), Ordered Sets, Reidel, Dordrecht, Boston, 1982.

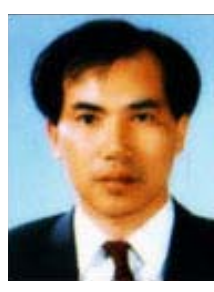

Yong Chan Kim He received the M.S and Ph.D. degrees in Department of Mathematics from Yonsei University, in 1984 and 1991, respectively. From 1991 to present, he is a professor in Department of Mathematics, Gangneung-Wonju University .His research interests are fuzzy logic and fuzzy topology.

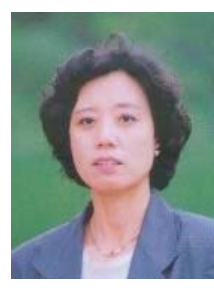

Jung Mi Ko She received the M.S and Ph.D. degrees in Department of Mathematics from Yonsei University, in 1983 and 1988, respectively. From 1988 to present, she is a professor in Department of Mathematics, Gangneung-Wonju University. Her research interests are fuzzy logic. 